\title{
The extremely high-velocity molecular outflow in IRAS 20126+4104
}

\author{
M. Lebrón ${ }^{1}$, H. Beuther ${ }^{2,3}$, P. Schilke ${ }^{4}$, and Th. Stanke ${ }^{4,5}$
}

\author{
1 Arecibo Observatory, HC 03 Box 53995, Arecibo 00612, Puerto Rico \\ e-mail: mlebron@naic.edu \\ 2 Harvard-Smithsonian Center for Astrophysics, 60 Garden Street, MS 78 Cambridge, MA 02138, USA \\ 3 Max-Planck-Institute for Astronomy, Königstuhl 17, 69117 Heidelberg, Germany \\ ${ }^{4}$ Max-Planck-Institut für Radioastronomie, Auf dem Hügel 69, 53121 Bonn, Germany \\ 5 University of Hawaii, Institute for Astronomy, 2680 Woodlawn Drive, Honolulu, Hawaii 96822-1897, USA
}

Received 17 August 2004 / Accepted 7 October 2005

\section{ABSTRACT}

New sensitive CO(2-1) observations of the massive star-forming region IRAS 20126+4104 reveal a high-velocity gas component that has not been observed before. Comparing the morphologies of the high-velocity $\mathrm{CO}$ and the molecular jet traced by $\mathrm{H}_{2}$ and $\mathrm{SiO}$, we found various orientations projected on the plane of the sky that could be interpreted in terms of jet precession. The outflow shows a steepening mass spectrum with increasing flow velocities. The high-velocity gas in IRAS 20126+4104 contributes significantly to the energy and momentum of the flow.

Key words. stars: formation - ISM: jets and outflows - ISM: individual objects: IRAS 20126+4104

\section{Introduction}

The presence of outflows is an indicator of protostars in a very early evolutionary stage. Although molecular outflows are ubiquitous phenomena in high-mass star formation (Shepherd \& Churchwell 1996; Zhang et al. 2001; Beuther et al. 2002), their formation and evolution are not properly understood.

In this paper, we present sensitive broadband observations of the ${ }^{12} \mathrm{CO}(2-1)$ line of the high-mass star-forming region IRAS $20126+4104$, complemented by an IR $\mathrm{H}_{2}$ image. This source shows a well-defined bipolar outflow (Shepherd et al. 2000; Wilking et al. 1990) that is very likely driven by a collimated jet (Cesaroni et al. 1999). Previous observations of the large scale molecular outflow have shown a north-south orientation (Wilking et al. 1990; Shepherd et al. 2000). Furthermore, $\mathrm{H}_{2}$ knots are found in the region indicating shocked gas in the outflow (Ayala et al. 1998; Shepherd et al. 2000). The "S" distribution of the $\mathrm{H}_{2}$ knots seems to follow the boundaries of the CO-lobes suggesting that the jet in IRAS 20126+4104 is precessing (Shepherd et al. 2000). In this work, we explore the high-velocity gas of the large scale outflow in IRAS 20126+4104 and compare it with other observations.

\section{Observations}

CO data. IRAS $20126+4104$ was observed in the $J=$ 2-1 transition of ${ }^{12} \mathrm{CO}$ in March 2002 at the IRAM $30 \mathrm{~m}$ telescope using the 9 beam receiver HERA. At $230.5 \mathrm{GHz}$, the resolution of the $30 \mathrm{~m}$ telescope is approximately $11^{\prime \prime}$. We made an on-the-fly (otf) map of $150^{\prime \prime} \times 200^{\prime \prime}$ centered on $\alpha_{2000}=20^{\mathrm{h}} 14^{\mathrm{m}} 26.0^{\mathrm{s}}, \delta_{2000}=+41^{\circ} 13^{\prime} 32^{\prime \prime}$. The map was done with a sampling interval of $4^{\prime \prime}(\sim$ Nyquist sampling), and the dump time was $2 \mathrm{~s}$ per position. The region was scanned twice in perpendicular directions to reduce the scanning effects in the map. The VESPA (VErsatile SPectral Assembly) autocorrelator was used with a bandwidth of $320 \mathrm{MHz}$ and a channel resolution of $1.25 \mathrm{MHz}$, corresponding to $1.6 \mathrm{~km} \mathrm{~s}^{-1}$ at $230.5 \mathrm{GHz}$. Each HERA beam is connected to one independent part of VESPA, and the LSR velocity of the system is $-3.5 \mathrm{~km} \mathrm{~s}^{-1}$. The spectra were reduced and analyzed in CLASS, and the mapping was done with GRAPHIC, both part of the GILDAS data reduction package of the Observatoire de Grenoble and IRAM. The two perpendicular sets of otf maps were averaged and de-striped of scanning effects by using the Emerson \& Gräve (1988) plait algorithm adapted to spectral line observations (see Hoernes 1997). The intensity scale of the data is in units of antenna temperature $\left(T_{A}^{*}\right)$. The emission beam efficiency for HERA is 0.52 , and the rms per channel was $0.17 \mathrm{~K}$.

$\mathbf{H}_{2}$ image. Sensitive wide-field near infrared $K$-Band images of IRAS 20126+4104 were obtained on June 7th 2001 using Omega Prime on the Calar Alto $3.5 \mathrm{~m}$ telescope in Spain. Images were taken through a $2.12 \mu \mathrm{m}$ narrow band filter $\left(\mathrm{H}_{2}\right.$ line filter) and a broad band $K^{\prime}$ filter for continuum subtraction. The camera provides a field of view of about $6.7 \times$ $6.7 \mathrm{arcmin}$ at a pixel scale of $0.4 \mathrm{arcsec} / \mathrm{pixel}$. The total field 


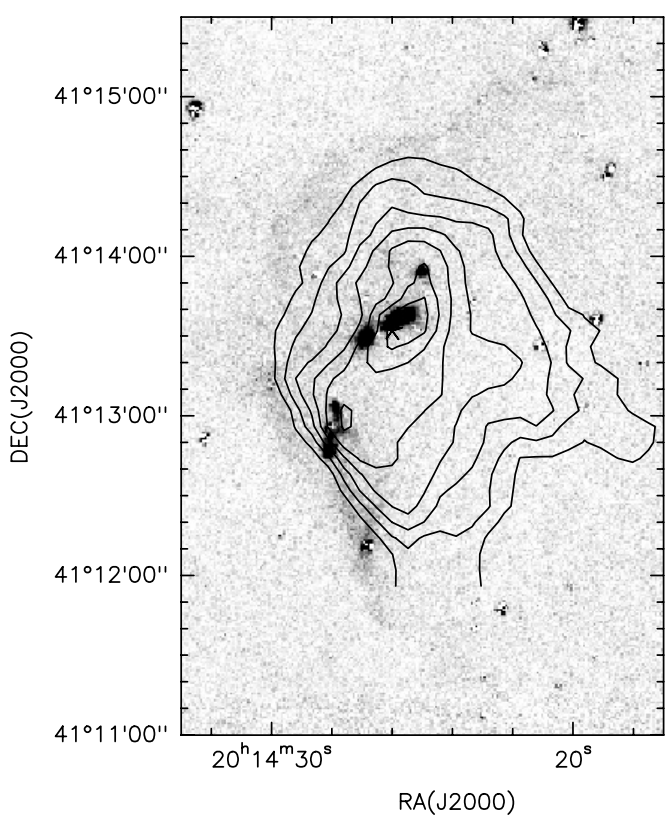

Fig. 1. $\mathrm{CO}(2-1)$ gas emission (contours) and $\mathrm{H}_{2}$ emission (background image). The $\mathrm{CO}$ image was obtained integrating the emission from -7.7 to $-2.8 \mathrm{~km} \mathrm{~s}^{-1}$. The contour levels range from 10 to $90 \%$ (in step of $10 \%$ ) of the peak integrated emission of $79.1 \mathrm{~K} \mathrm{~km} \mathrm{~s}^{-1}$.

covered is about $8.7 \times 8.7$ arcmin. Data reduction followed standard procedures (for details see Stanke et al. 2006). The seeing in the final co-added images was about 1.5 arcsec.

\section{The high-velocity flow}

In Fig. 1 we present an integrated $\mathrm{CO}$ map of the molecular cloud superimposed on the $\mathrm{H}_{2}$ image to outline the $\mathrm{H}_{2}$ emission. A new $\mathrm{H}_{2}$ feature appears as a faint glow outlining the eastern surface of the cloud harboring IRAS 20126+4104. This emission is most likely due to fluorescent emission from molecular gas being illuminated by UV emission from the east.

It is found that the molecular outflow in IRAS 20126+4104 extends at least up to $-58 \mathrm{~km} \mathrm{~s}^{-1}$ in the blue lobe and $+55 \mathrm{~km} \mathrm{~s}^{-1}$ in the red lobe (see Figs. 2-4). Such high-velocity outflow has so far been observed only in very few sources (e.g. L1448: Bachiller \& Tafalla 2000; NGC 2023mm1: Sandell et al. 1999). Figure 2 shows channel maps (averaged over $12-16 \mathrm{~km} \mathrm{~s}^{-1}$ each) of the $\mathrm{CO}(2-1)$ emission of the outflow. The greyscale shows the $\mathrm{H}_{2} 2.12 \mu \mathrm{m}$ line image. Although the sensitivity, area coverage, and angular resolution of the new $\mathrm{H}_{2}$ image are significantly better than in published images, no new $\mathrm{H}_{2}$ shock features attributable to outflow activity were found in the region.

Figure 3 includes the spectra from two different positions of the outflow in the red and blue lobes. The very broad wings are clearly visible. For a better understanding of the velocity distribution, a position-velocity $(p-v)$ diagram is shown in Fig. 3. The direction of the $p-v$ plot is indicated with a diagonal line in Fig. 2h. The outflow shows two peaks of maximal velocity in the red wing and one in the blue. Multiple peaks in $p-v$ diagrams of a single outflow can be interpreted as episodic events in the flow (Arce \& Goodman 2001). From the position-velocity diagram, it is possible to see an increase in the maximal velocity as the distance to the central source increases. This displacement is also clear from the channel maps shown in Fig. 2 (panels a to d), where higher velocity gas is located farther away from the central source. Comparing that with the model of a conical outflow with the different velocity distributions of Cabrit \& Bertout $(1986,1990)$, the outflow in 20126 is consistent with the accelerating outflow, where the bulk of the emission is at lower velocities (and near the central source) and the maximal velocity increases as the distance to the central source increases. One possibility for accelerating the flow is that due to momentum conservation in a medium with a density gradient, the flow is accelerated toward the low density gas (Bachiller \& Tafalla 2000).

The outflow in 20126 also shows more compact emission as the velocity increases, and the high-velocity gas seems to be in the wake of the lower velocity gas. Linear displacement of the emission as the velocity increases has been found in sources like L1551 (Bachiller \& Tafalla 2000) and G192.16 (Shepherd et al. 1998), where the low velocity gas shows a shell-like structure and is closer to the central source, while the high velocity gas is farther away (from the continuum peak), more compact, and aligned with the flow axis. This morphology seems to be present in flows from low-mass up to early-B stars. But higher spatial resolution of the outflow in 20126 is necessary to better distinguish the distribution of the high-velocity gas.

\section{Discussion}

\subsection{Multiple outflows or precession}

A well-collimated molecular jet, detected in $\mathrm{SiO}$ (Cesaroni et al. 1999) and $\mathrm{H}_{2}$ (Ayala et al. 1998; Shepherd et al. 2000), is located at the heart of the IRAS 20126+4104 source. Cesaroni et al. (1999) explain the $\mathrm{SiO}$ emission as a molecular jet with an opening angle $\theta \sim 21^{\circ}$ that is oriented almost parallel to the plane of the sky with an inclination angle $\phi \sim 9^{\circ}$ (angle of the flow with respect to the plane of the sky). On larger spatial scales, the molecular outflow shows a different shape. The lower velocity gas is oriented north-south (Wilking et al. 1990), while the higher velocity flow is oriented northwest-southeast (Shepherd et al. 2000). The observations presented here show a very high-velocity flow inclined even more to the northwestsoutheast. It is now worthwhile to examine how the very highvelocity gas is related to the previously found flow and collimated jet.

Figure 4 compares the high-velocity $\mathrm{CO}\left(v>| \pm 31| \mathrm{km} \mathrm{s}^{-1}\right)$ with the outflow in $\mathrm{HCO}^{+}$, the jet in $\mathrm{SiO}$ (Cesaroni et al. 1997), and the $\mathrm{H}_{2}$ line emission. The velocity range covered by the $\mathrm{HCO}^{+}$flow and $\mathrm{SiO}$ jet are $v<| \pm 21| \mathrm{km} \mathrm{s}^{-1}$. The direction of the $\mathrm{SiO}$ jet is indicated with a thick line. The main axis of the $\mathrm{HCO}^{+}$flow (thin contours) follows the $\mathrm{SiO}$ jet but not the direction of high-velocity $\mathrm{CO}$ (thick contours). The $\mathrm{HCO}^{+}$flow and $\mathrm{SiO}$ jet are misaligned by $\sim 30^{\circ}$ (projected on the plane of the sky) with the high-velocity $\mathrm{CO}$ outflow. There is no evidence of an $\mathrm{SiO}$ jet in the direction of the high-velocity $\mathrm{CO}$. Low-level emission of $\mathrm{HCO}^{+}$shows some elongation in the direction of high-velocity CO (see Fig. 4). 


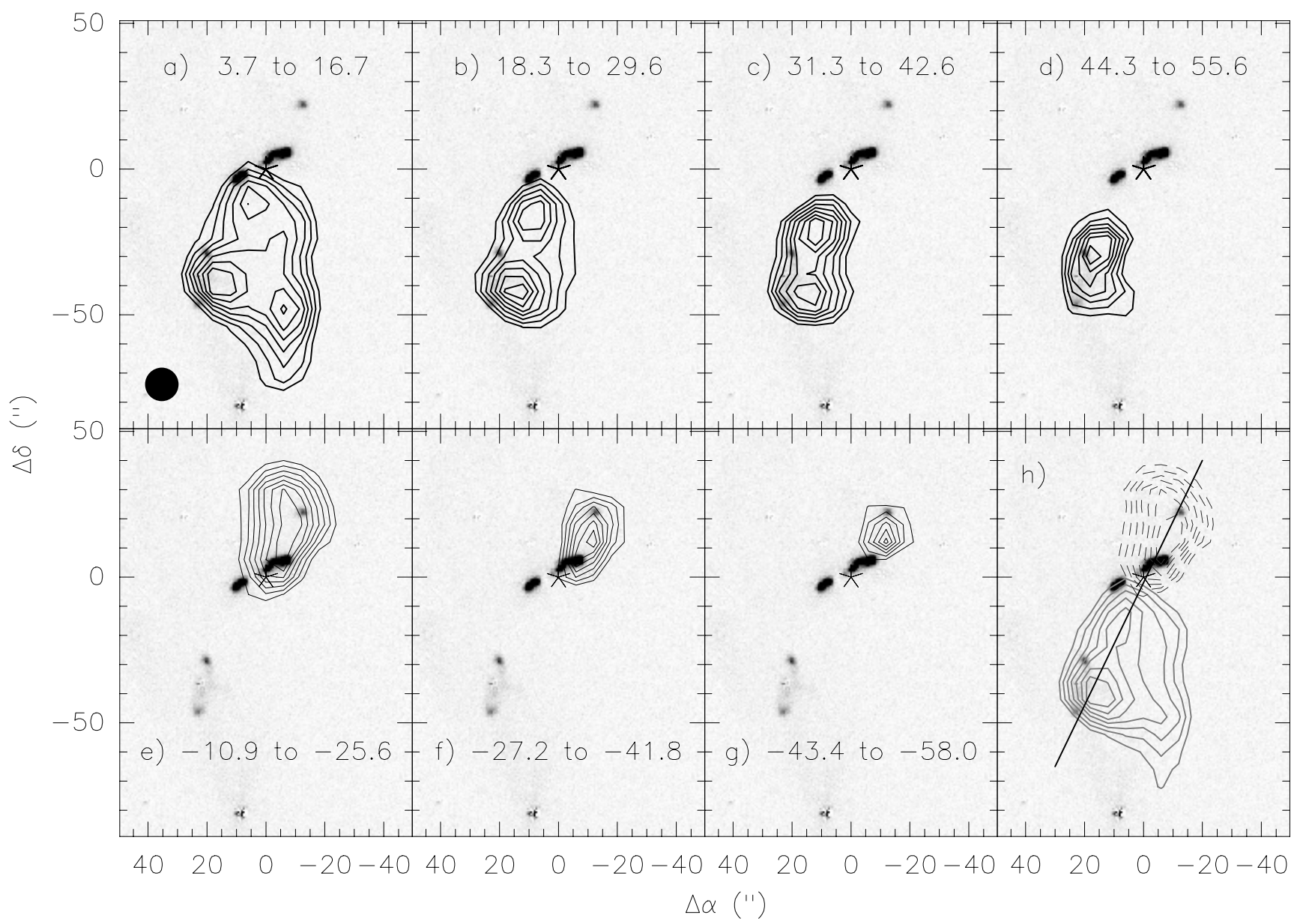

Fig. 2. Velocity integrated channel maps of the ${ }^{12} \mathrm{CO}(2-1)$ emission in the outflow. The upper panels (a) to d)) show the red lobe, while the lower panels (e) to $\mathbf{g}$ )) show the blue lobe. The velocity range is indicated in each panel. Notice that not all velocity integration ranges have the same width. The different ranges were chosen to put channels together with similar $\mathrm{CO}(2-1)$ emission structure. Panel h) shows the total outflow integrated between 3.7 and $55.6 \mathrm{~km} \mathrm{~s}^{-1}$ in the red lobe (full-line contours) and between -58.0 and $-10.9 \mathrm{~km} \mathrm{~s}^{-1}$ in the blue lobe (dashed-line contours). Each map's center is indicated by a star symbol. The gray scale presents the $\mathrm{H}_{2}$ emission, and the $11^{\prime \prime}$ beam is shown at the bottom left corner of panel a). The contour levels range from 30 to $90 \%$ (in steps of $10 \%$ ) of the peak-integrated emission in each panel. The peak values for panels a) to g) are 53.6, 18.9, 12.6, 7.3, 40.0, 9.6, and $6.5 \mathrm{~K} \mathrm{~km} \mathrm{~s}^{-1}$, respectively, and for the red and blue lobes in panel $\mathbf{h}$ ) 85.7 and $50.5 \mathrm{~K} \mathrm{~km} \mathrm{~s}^{-1}$, respectively.

Why is there no $\mathrm{SiO}$ emission in the direction of the highvelocity $\mathrm{CO}$ ? One possible explanation is that the outflow has evolved enough that the $\mathrm{SiO}$ molecules are oxidized or retrapped on the dust grains. The time scale for re-trapping the $\mathrm{SiO}$ in dust grains is $\sim 10^{4}$ years (Pineau des Forets et al. 1997). If we consider the dynamical time (4800 years) of the high-velocity $\mathrm{CO}$ as an indication of the age of the flow, then $\mathrm{SiO}$ emission should still be seen. But in the case of 20126, the dynamical time is only a lower limit to the flow age because, as we discussed before, the flow seems to be accelerating. The real age of the flow could be greater than $10^{4}$ years, and the $\mathrm{SiO}$ could already be converted to $\mathrm{SiO}_{2}$ molecules (that don't have a detectable line) or re-aggregated onto dust (Pineau des Forets et al. 1997).

The misalignment of the $\mathrm{HCO}^{+}$outflow and the $\mathrm{SiO}$ jet, with the high-velocity $\mathrm{CO}$ flow, might be readily explained by a gradual change of outflow direction (e.g. precession). Based on the spatial coincidence of $\mathrm{H}_{2}$ knots distributed in an "S" shape following the edges of the low-velocity CO flow and on the lack of evidence of other embedded sources in the region, Shepherd et al. (2000) favor the precession hypothesis. We re-evaluate this possibility taking the high-velocity gas into account. The fact that no SiO-shocked emission is observed in the direction of the high-velocity $\mathrm{CO}$ suggests that the highvelocity $\mathrm{CO}$ is older than the $\mathrm{SiO}$ emission. If the jet is precessing, it then must be located first in the direction of the high-CO and moved at least by $30^{\circ}$ to its present position where it is exciting the $\mathrm{SiO}$ emission. Because there is no evidence of shocks or flows in angles between the $\mathrm{SiO}$ jet and high-CO, it is most likely that the precessing jet is also a time variable jet. Shepherd et al. (2000) discuss three different scenarios that can generate dramatic precession in the jet, but only the anisotropic accretion events can also be considered as a time variable scenario. During the collision of a low mass condensation with a massive star accretion disk in an anisotropic accretion event, it is possible to re-orient the disk by up to $30^{\circ}$.

Another potential explanation for the misalignment of the molecular jet with the high-velocity $\mathrm{CO}$ is to consider that they 


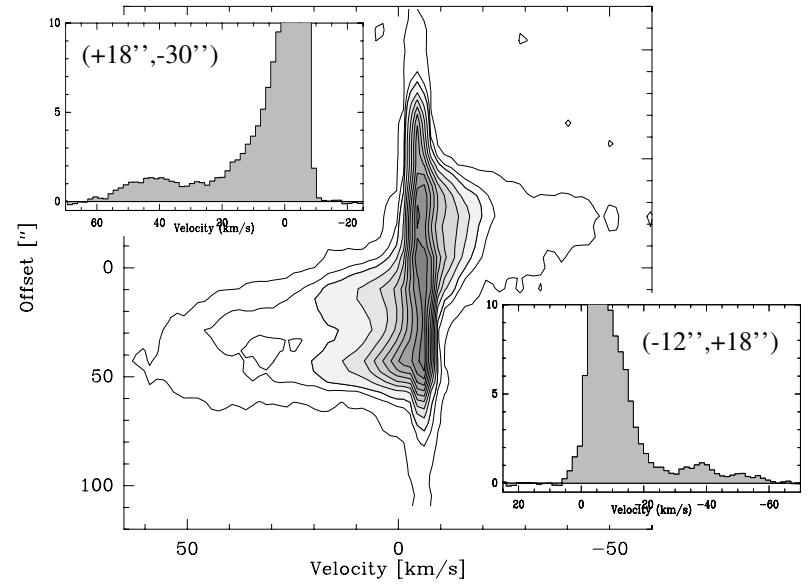

Fig. 3. Position-velocity diagram of the outflow in IRAS 20126+4104. The direction of the slice from where the $p-v$ was done is indicated in Fig. $2 \mathrm{~h}$ with a diagonal line. In the upper-left and lower-right corners, we included the $\mathrm{CO}$ spectrum from two positions (indicated in parenthesis) in the red and blue lobes, respectively.

are produced by different sources. The high-velocity CO flow has a larger dynamical age than the $\mathrm{SiO}$ jet. As discussed before, the absence of $\mathrm{SiO}$ emission in the direction of the highvelocity $\mathrm{CO}$ could indicate different evolutionary stages for both flows. In this case, the high-velocity $\mathrm{CO}$ flow will come from a more evolved massive $\mathrm{YSO}$, while the $\mathrm{SiO}$ and $\mathrm{H}_{2}$ jets will come from a younger YSO. So far, only two radio continuum sources (Hofner et al. 1999) have been identified in the region. They are elongated in the direction of the molecular jet and are separated by $\sim 1^{\prime \prime}$. The continuum source north seems to be related to the mm-continuum emission and $\mathrm{H}_{2} \mathrm{O}$ masers, while the source south does not show any maser emission that could be used as an indication of star formation (Hofner et al. 1999). Recently, Sridharan et al. (2005) show a double infrared source at the center of IRAS $20126+4104$, which could in principle account for multiple outflows in the region.

However, the double-source also fits well into the precession interpretation for this outflow (Shepherd et al. 2000). Also considering the rather regular shift from the low-velocity northsouth flow in the direction of the high-velocity north-west south-east flow (Fig. 2), among with the spatial correlation of the high-velocity $\mathrm{CO}$ gas with the low-level $\mathrm{HCO}^{+}$emission, on the one hand, and the $\mathrm{HCO}^{+}$and $\mathrm{SiO}$ emission, on the other hand (Fig. 4), we favor the precession hypothesis to explain the IRAS 20126+4104 outflow. Then the idea is that the jet was first oriented in the direction of the low-velocity $\mathrm{CO}$ emission, then moved to the direction of the high-velocity $\mathrm{CO}$ outflow, and last but not least moved to its actual orientation, leaving behind the low level emission in the $\mathrm{HCO}^{+}$outflow and the high-velocity $\mathrm{CO}$ as evidence. The fact that the $\mathrm{H}_{2}$ knots follow the edges of the high-velocity flow also suggest a relation with the $\mathrm{CO}$ flow, but to establish that relation, a detailed study of the $\mathrm{H}_{2}$ knots is necessary. The Northwest $\mathrm{H}_{2}$ knot is an emission line object (Ayala et al. 1998) that also shows blueshifted $\mathrm{SiO}$ emission (from Cesaroni $\mathrm{SiO}$ data) indicating its possible shock origin. For the southern $\mathrm{H}_{2}$ knots, neither $\mathrm{SiO}$ information nor FIR spectroscopy is available; in fact, the

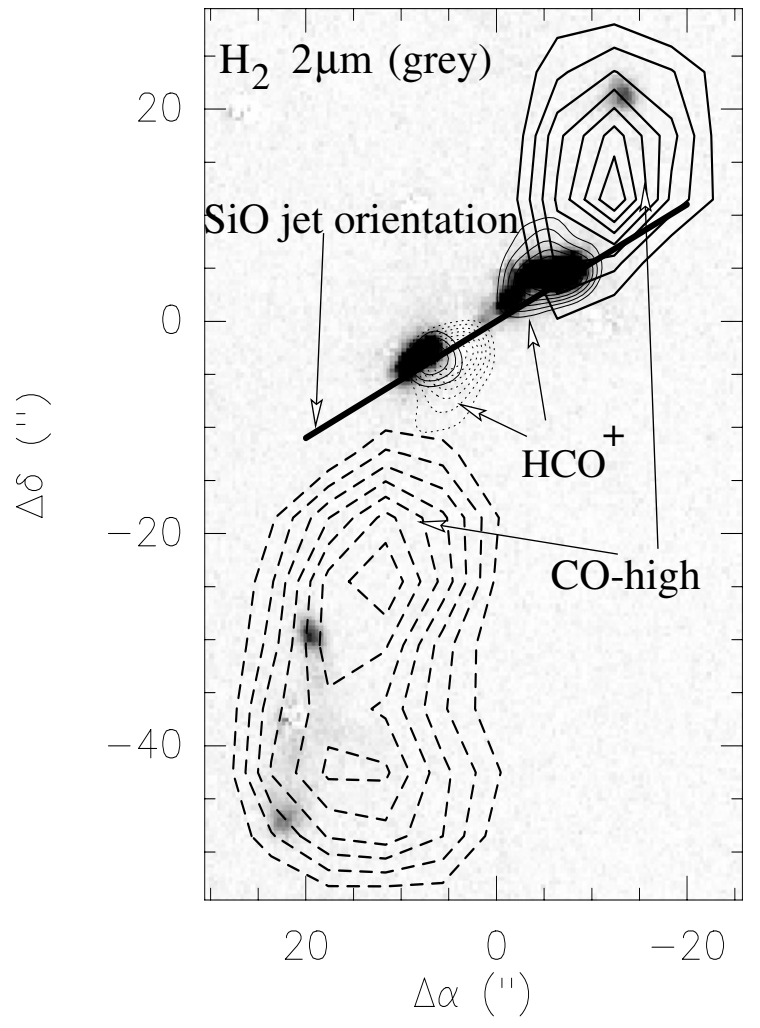

Fig. 4. Map of the high-velocity ${ }^{12} \mathrm{CO}(2-1)$ emission (dark-line contours) showing the distribution of the outflow gas. The continuous lines represent the blue emission integrated from -58.0 to $-33.7 \mathrm{~km} \mathrm{~s}^{-1}$, while the dashed lines represent the red emission integrated from 31.3 to $55.6 \mathrm{~km} \mathrm{~s}^{-1}$. The contour levels range from 30 to $90 \%$ (in steps of $10 \%$ ) of the peak intensity of 11.6 and $19.0 \mathrm{~K} \mathrm{~km} \mathrm{~s}^{-1}$ for the blue and red lobes, respectively. The thin line contours are of the outflow in $\mathrm{HCO}^{+}(1-0)$ (from Cesaroni et al. 1997). In the background is the $\mathrm{H}_{2}$ image.

$\mathrm{H}_{2}$ morphology at low intensity levels might suggest a relation to a star located in between the two knots rather than toward the CO outflow driven by 20126 itself. However, as pointed out by Shepherd et al. (2000), the central star has near-infrared colors consistent with a foreground object and no infrared excess. Furthermore, no mm continuum emission is detected there either. Hence, it is unlikely that this source drives another outflow in the region.

\subsection{Masses and energetics in the outflow}

Opacity-corrected $\mathrm{H}_{2}$ column densities can be calculated by assuming ${ }^{13} \mathrm{CO} /{ }^{12} \mathrm{CO}$ line wing ratios. Choi et al. (1993) found an average ${ }^{13} \mathrm{CO} /{ }^{12} \mathrm{CO} 2-1$ ratio of 0.1 , which we adopt for the calculations of the outflow parameters integrated over the whole high-velocity gas. Variations of this ratio with velocity will be discussed below. The assumed temperature is $30 \mathrm{~K}$, and the $\mathrm{H}_{2} /{ }^{13} \mathrm{CO}$ ratio is $89 \times 10^{4}$. The outflow was integrated for velocities that were greater than $\pm 7 \mathrm{~km} \mathrm{~s}^{-1}$ from the LSR. That excludes the very low-velocity flow. Based on the previously derived inclination angles for the $\mathrm{SiO}$ jet of $\sim 10^{\circ}$ (Cesaroni et al. 1999) and for the low-velocity CO outflow of $\sim 45^{\circ}$ (Shepherd et al. 2000), we assumed an inclination angle of $20^{\circ}$ 
Table 1. Outflow properties.

\begin{tabular}{lc}
\hline \hline Parameter & Value \\
\hline$\Delta v_{b}\left(\mathrm{~km} \mathrm{~s}^{-1}\right)$ & $(-58,-11)$ \\
$\Delta v_{r}\left(\mathrm{~km} \mathrm{~s}^{-1}\right)$ & $(4,55)$ \\
$v_{\mathrm{LSR}}\left(\mathrm{km} \mathrm{s}^{-1}\right)$ & -3.5 \\
$N_{b}\left(\mathrm{~cm}^{-2}\right)$ & $1.25 \times 10^{21}$ \\
$N_{r}\left(\mathrm{~cm}^{-2}\right)$ & $1.99 \times 10^{21}$ \\
$M_{b}\left(M_{\odot}\right)^{a}$ & 4.2 \\
$M_{r}\left(M_{\odot}\right)^{a}$ & 12.1 \\
$M_{\text {total }}\left(M_{\odot}\right)^{a}$ & 16.3 \\
Momentum $\left(M_{\odot} \mathrm{km} \mathrm{s}^{-1}\right)^{b}$ & 1490 \\
Energy $(\operatorname{erg})^{b}$ & $1.3 \times 10^{48}$ \\
Kinematical timescale $\left(\mathrm{yr}^{b}\right.$ & 4800 \\
Mass entrainment rate $\left(M_{\odot} \mathrm{yr}^{-1}\right)^{b}$ & $3.4 \times 10^{-3}$ \\
Mechanical force $\left(M_{\odot} \mathrm{yr}^{-1} \mathrm{~km} \mathrm{~s}^{-1}\right)^{b}$ & $3.1 \times 10^{-1}$ \\
Mechanical luminosity $\left(L_{\odot}\right)^{b}$ & 2300 \\
\hline
\end{tabular}

${ }^{a}$ Distance to the source $1.7 \mathrm{kpc}$ (Shepherd et al. 2000).

${ }^{b}$ An inclination angle of $20^{\circ}$ in the flow was assumed.

for the high-velocity outflow. To derive the kinematical parameters of the integrated high-velocity outflow, we used the maximum observed velocities corrected for the inclination angle (see Cabrit \& Bertout 1990, caution is necessary because they define the inclination angle differently as the angle between the outflow axis and the line of sight.). The kinematical parameters calculated in this way are always upper limits. The mass calculations are estimated to be correct approximately within a factor 2, whereas their kinematical parameters have larger errorbars on the order of a factor 10. More details on the assumptions, calculations, and error estimates can be found in Cabrit \& Bertout (1990) and Beuther et al. (2002). The derived values are compiled in Table 1.

Comparing the total outflow mass of $53 M_{\odot}$ determined by Shepherd et al. (2000) (they integrated the flow emission from $\pm 2.7 \mathrm{~km} \mathrm{~s}^{-1}$ from the LSR) with the $16 M_{\odot}$ presented here, we find that most of the mass is contained in the low-velocity gas. In contrast to this, energetic quantities - like the energy or the mass-entrainment rate - can significantly increase when taking the high-velocity gas into account. Comparing the derived parameters in IRAS 20126+4104 with the results by Beuther et al. (2002), we see that the outflow mass in IRAS 20126+4104 is about average, while energetic parameters - e.g., the energy or the mass-entrainment rate - are at the upper end of the observed parameter range. The low mass we derived for IRAS $20126+4104$, is due to the fact that we used only highvelocity gas, while Beuther et al. 2002 used on average lower velocity emission as well. In contrast, obtaining high kinematic and energetic parameters using only high-velocity emission, stresses the importance of the high-velocity gas for the total energy budget of the region.

For a direct comparison of our results and the previously derived kinematical parameters by Shepherd et al. (2000) for IRAS 20126+4104, one has to keep in mind that Shepherd et al. (2000) used slightly different assumptions. They calculated the opacity as a function of the velocity, while we assumed optically thick ${ }^{12} \mathrm{CO}$ for the complete outflow. They used the intensity weighted velocity in the time scale, while we used the maximum velocity (dynamical time). They assumed an inclination angle and temperature of $45^{\circ}$, and $44 \mathrm{~K}$, respectively, while we assumed $20^{\circ}$, and $30 \mathrm{~K}$, respectively. All these assumptions systematically result in lower kinematical parameters than we obtained. As outlined in Cabrit \& Bertout (1990) the differences should be on the order of a factor 10. While the kinetic energy we derived is only a factor 20 higher than the value by Shepherd et al. (2000), which is approximately within the error bars, the mechanical force is a factor 50 higher, and the mechanical luminosity even approximately a factor 400 . These last differences are above the estimated error budget and show that the high-velocity gas is important for an accurate determination of the kinematic parameters of molecular outflows.

Due to the wide band used for this observation and the better sensitivity of the data (rms of $170 \mathrm{mK}$ per channel), compared with the CO observations of Shepherd et al. (2000) (rms of $300 \mathrm{mK}$ per channel), we were able to detect the very high-velocity gas of the flow. That high sensitivity allows a detailed study of the variation of the outflow parameters with increasing velocity. While the absolute values of the masses and energies are very uncertain (Cabrit \& Bertout 1990; Beuther et al. 2002), the relative accuracy between the different velocity channels should be fairly good. The largest uncertainty arises from the unknown opacity change through the line wings: we apply an opacity correction throughout the whole line wing based on an average ${ }^{12} \mathrm{CO} /{ }^{13} \mathrm{CO} 2 \rightarrow 1$ line ratio of 10 (see Choi et al. 1993), which corresponds to $\tau\left({ }^{12} \mathrm{CO}\right)=9$. But Choi et al. (1993) also show that the opacity can decrease with higher velocities, and at the high-velocity end of our analysis, the derived parameters are likely to be just an upper limit to the real masses and energies. Therefore we redid the mass and energy calculations over the whole line wing assuming ${ }^{12} \mathrm{CO}$ to be optical thin. Figure 5 presents the masses and energies for both calculations versus the observed velocity.

The mass-velocity relation in the red wing of IRAS $20126+4104$ can be separated into a low-velocity part $\left(v_{\text {low }}<20 \mathrm{~km} \mathrm{~s}^{-1}\right)$, an intermediate-velocity part $(20<$ $\left.v_{\text {im }}<40 \mathrm{~km} \mathrm{~s}^{-1}\right)$, and a high-velocity part $\left(v_{\text {high }}>40 \mathrm{~km} \mathrm{~s}^{-1}\right)$. Each part can be fitted well by a power law $\left(M \propto v^{-\gamma}\right)$, and $\gamma$ increases with increasing velocity (see Fig. 5). The range of observed $\gamma$ spans a similar regime to the outflows compiled by Richer et al. (2000). As lower opacities for higher velocities only reduce those masses, the steepening to the outside is real. Similar findings have been reported in the literature (Bachiller \& Tafalla 2000; Richer et al. 2000), but this contrasts with recent observations by Molinari et al. (2002), who find a flattening at higher velocities, based on their $\mathrm{HCO}^{+}$observations. They argue that the sample compiled by Richer et al. (2000) could be biased to older sources, and that the younger ones might be flatter. This suggestion has to be checked with a larger sample of sources, but we note that IRAS $20126+4104$ is part of the initial sample compiled by Molinari et al. (1996), and that it is a very young source. There might be more consistency problems between the $\mathrm{HCO}^{+}$observations and usual $\mathrm{CO}$ observations than believed so far. A reasonable fit for the mass-velocity distribution in the 

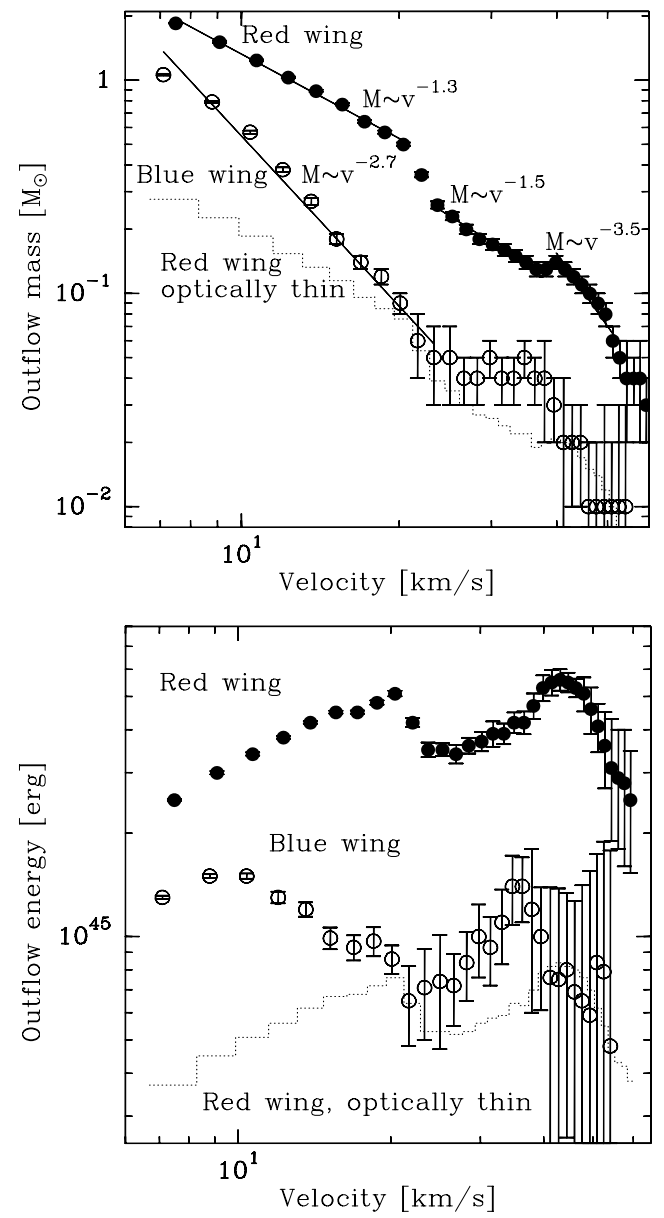

Fig. 5. Mass-velocity distribution (upper panel) and energy-velocity distribution (lower panel) of the outflow. The dotted line in each panel are the mass and energy values assuming optically thin ${ }^{12} \mathrm{CO}$. For the energy distribution an inclination angle of $20^{\circ}$ in the flux was assumed.

blue wing is only possible in the regime of $v_{\text {low }}<20 \mathrm{~km} \mathrm{~s}^{-1}$, but at higher velocities the flow appears to be flatter.

Assuming constant opacities for the whole wing emission, the energy per velocity bin is approximately the same order of magnitude throughout the whole velocity range. In contrast, if the ${ }^{12} \mathrm{CO}$ emission turns optically thin at extremely high velocities, the energy budget of the whole outflow can be dominated by the lower velocity gas. Nevertheless, the data show that a significant fraction of the total energy within molecular outflows is contained in gas at high velocities. Considering that many outflow observations are sensitivity limited, their calculated energies might only be lower limits of the total energy released by the molecular outflow. For more accurate estimates and a real understanding of the energetics in molecular outflows at high velocities, high-sensitivity observations of optically thin lines are still necessary.

\section{Conclusions}

The outflow in IRAS 20126+4104 exhibits high-velocity gas detected in ${ }^{12} \mathrm{CO}(2-1)$ out to $\pm 50 \mathrm{~km} \mathrm{~s}^{-1}$. Comparing the highvelocity $\mathrm{CO}$ with the $\mathrm{SiO}$ jet observed in the region (Cesaroni et al. 1999), we found that the very high-velocity CO flow is inclined $\sim 30^{\circ}$ with respect to the $\mathrm{SiO}$ jet. This discrepancy supports the idea of precession in the jet proposed by Shepherd et al. (2000). However multiple flows produced by independent sources in the region cannot be discarded.

Because of the high sensitivity of the data we are able to study the mass-velocity distribution of the flow up to $50 \mathrm{~km} \mathrm{~s}^{-1}$. We found steeper slopes as the velocity increases consistently with results obtained by Bachiller \& Tafalla (2000), and Richer et al. (2000), but not with the study of Molinari et al. (2002), who found flat mass-velocity distributions based on $\mathrm{HCO}^{+}$observations. The high-velocity gas contributes significantly to the total energy of the molecular outflow.

Acknowledgements. We like to thank the referee Debra Shepherd, whose detailed suggestions and comments improved the manuscript significantly. We thank Dr. R. Cesaroni for providing us with the $\mathrm{HCO}^{+}$and $\mathrm{SiO}$ maps. H.B. acknowledges financial support by the Emmy-Noether-Program of the Deutsche Forschungsgemeinschaft (DFG, grant BE2578/1).

\section{References}

Arce, H. G., \& Goodman, A. A. 2001, AJ, 55, L171

Ayala, S., Curiel, S., Raga, A. C., Noriega-Crespo, A., \& Salas, L. 1998, A\&A, 332, 1055

Bachiller, R., \& Tafalla, M. 2000, Bipolar Molecular Outflows, in The Origins of Stars and Planetary Systems, ed. C. J. Lada, \& N. D. Kylafis (Kluwer Academic Press), 227

Beuther, H., Schilke, P., Sridharan, T. K., et al. 2002, A\&A, 383, 892

Cabrit, S., \& Bertout, C. 1986, ApJ, 307, 313

Cabrit, S., \& Bertout, C. 1990, ApJ, 348, 530

Cesaroni, R., Felli, M., Jenness, T., et al. 1999, A\&A, 345, 949

Cesaroni, R., Felli, M., Testi, L., Walmsley, M. C., \& Olmi, L. 1997, A\&A, 325, 725

Choi, M., Evans, N. J. II, \& Jaffe, D. T. 1993, ApJ, 417, 624

Emerson, D. T., \& Gräve, R. 1988, A\&A, 190, 353

Hoernes, P. 1997, Thesis, Universität Bonn

Hofner, P., Cesaroni, R., Rodríguez, L. F., \& Martí, J. 1999, A\&A, 345, L43

Langer, W., \& Penzias, A. 1990, ApJ, 357, 477

Molinari, S., Brand, J., Cesaroni, R., \& Palla, F. 1996, A\&A, 308, 573

Molinari, S., Testi, L., Rodriguez, L. F., \& Zhang, Q. 2002, ApJ, 570, 758

Pineau des Forets, G., Flower, D. R., \& Chieze, J.-P. 1997, The Physical and Chemical Effects of C-Shocks in Molecular Outflows, in Herbig-Haro Flows and the Birth of Low Mass Stars, ed. V. Mannings, A. P. Boss, \& S. S. Russell (Kluwer, Dordrecht), Proc. IAU Symp., 182, 199

Richer, J., Shepherd, D., Cabrit, S., Bachiller, R., \& Churchwell, E. 2000, Molecular Outflows from Young Stellar Objects, in Protostars \& Planets IV, ed. V. Mannings, A. P. Boss, \& S. S. Russell (The University of Arizona Press), 867

Sandell, G., Avery, L. W., Baas, F., \& 16 coauthors 1999, ApJ, 519, 236

Shepherd, D. S., \& Churchwell, E. 1996, ApJ, 457, 267

Shepherd, D. S., Yu, K. C., Bally, J., \& Testi, L. 2000, ApJ, 535, 833

Shepherd, D. S., Watson, A. M., Sargent, A. I., \& Churchwell, E. 1998 ApJ, 507, 861

Sridharan, T. K., Williams, S. J., \& Fuller, G. A. 2005, ApJ, 631, 73

Stanke, et al. 2006, in preparation

Wilking, B. A., Blackwell, J. H., \& Mundy, L. G. 1990, AJ, 100, 758

Zhang, Q., Hunter T. R., Brand, J., et al. 2001, ApJ, 552, 167 